\begin{tabular}{|c|c|}
\hline Title & Songbird tap dancing produces non-vocal sounds \\
\hline Author(s) & Ota, Nao; Gahr, Manfred; Soma, Masayo \\
\hline Citation & $\begin{array}{l}\text { Bioacoustics: the international journal of animal sound and its recording, 26(2), 161-168 } \\
\text { https://doi.org/10.1080/09524622.2016.1231080 }\end{array}$ \\
\hline Issue Date & 2017-05-04 \\
\hline Doc URL & http:/hdl.handle.net/2115/67112 \\
\hline Rights & $\begin{array}{l}\text { This is an A ccepted Manuscript of an article published by Taylor \& Francis in Bioacoustics on May 2017, available } \\
\text { online: http://www.tandfonline.com/10.1080/09524622.2016.1231080. }\end{array}$ \\
\hline Type & article (author version) \\
\hline Additional Information & There are other files related to this item in HUSCAP. Check the above URL. \\
\hline File Information & manuscript_HUSCAP.pdf \\
\hline
\end{tabular}

Instructions for use 


\section{Songbird tap dancing produces non-vocal sounds}

Nao Ota ${ }^{\mathrm{a}}$, Manfred Gahr ${ }^{\mathrm{b}}$, and Masayo Soma ${ }^{\mathrm{c}^{*}}$

${ }^{a}$ Biosystems Science Course, The Graduate School of Life Science, Hokkaido University, Kita 10 Nishi 8 Kita-ku, Sapporo, Hokkaido 060-0810, Japan

${ }^{b}$ Department of Behavioural Neurobiology, Max Planck Institute for Ornithology, Eberhard-Gwinner-Str. 82319 Seewiesen, Germany

${ }^{\mathrm{c}}$ Department of Biology, Faculty of Science, Hokkaido University, Kita 10 Nishi 8

Kita-ku, Sapporo, Hokkaido 060-0810, Japan

Nao Ota

Manfred Gahr

Masayo Soma (masayo.soma@sci.hokudai.ac.jp) *Corresponding author

Tel: +81-11-706-2995

The affiliation where the research was conducted: Max Planck Institute for Ornithology, Eberhard-Gwinner-Str. 82319 Seewiesen, Germany 


\section{Abstract}

Vocalizations have been elucidated in previous songbird studies, whereas less attention has been paid to non-vocal sounds. In the bluecapped cordon-bleu (Uraeginthus cyanocephalus), both sexes perform courtship displays that are accompanied by singing and distinct body movements (i.e. dance). Our previous study revealed that their courtship bobbing includes multiple rapid steps. This behaviour is quite similar to human tap dancing, because it can function as both visual and acoustic signals. To examine the acoustic signal value of such steps, we tested if their high-speed step movements produce non-vocal sounds that have amplitudes similar to vocal sounds. We found that step behaviour affected step sound amplitude. Additionally, the dancing step sounds were substantially louder than feet movement sounds in a non-courtship context, and the amplitude range overlapped with that of song notes. These results support the idea that in addition to song cordon-bleus produce acoustic signals with their feet.

Keywords: multimodal communication, mutual courtship display, socially monogamous songbird, Estrildid finch, sonation, sound amplitude 


\section{Introduction}

Although birds generally rely on vocalization for acoustic communication, non-vocal sounds are known to be used in some species, such as manakins (Pipridae; Prum 1998; Bostwick and Prum 2003; Fusani et al. 2007), snipes (Gallinago coelestis; Bahr 1907), flappet larks (Mirafra rufocinnamomea; Norberg 1991), greater sage grouse (Centrocercus urophasianus; Koch et al. 2015), ruffed grouse (Bonasa umbellus; Garcia et al. 2012), hummingbirds (Trochilidae: Clark et al. 2011), woodpeckers (Picidae; Stark et al. 1998), oriental white storks (Ciconia boyciana; Eda-Fujiwara et al. 2004), and palm cockatoos (Probosciger aterrimus; Wood 1987). Generally, these species produce non-vocal sounds for courtship using their wings, tails, bills, or tools, and these non-vocal sounds are poorly understood in the context of acoustic communication evolution compared with vocalizations.

We recently found that both males and females of some songbird species also produce non-vocal sounds in addition to song (Ota et al. 2015; Soma and Mori 2015). For example, in Java sparrows (Lonchura oryzivora), which are socially monogamous songbirds without female song, both sexes produce bill-click sounds that are well-coordinated with male song (Soma and Mori 2015). These findings are rather surprising for at least two reasons. First, past studies of acoustic communication in songbirds have typically shed light on their vocalizations, because they are vocal learners and can acquire complex songs (Catchpole and Slater 2008). Therefore, non-vocal sounds in songbirds have been largely overlooked, although some songbirds were suggested to be able to produce non-vocal sounds (Clark and Prum 2015). Second, courtship displays to produce non-vocal sounds are usually considered to have evolved via strong sexual selection pressure on males of polygynous or lekking species (Andersson 1994; Prum 1998; Byers et al. 2010; Fusani and Schlinger 2012). Although a recent study found that vocalization is both widespread and ancestral in female songbirds (Odom et al. 2014), it is still unclear why and how non-vocal sounds evolved in males and females of some songbirds.

Our study species, the blue-capped cordon-bleu (Uraeginthus cyanocephalus), performs tap dance-like behaviour that is accompanied by distinct non-vocal sounds (Figure 1(a); Supplementary Video 1; Ota et al. 2015). Blue-capped cordon-bleus are socially monogamous songbirds, and both sexes perform elaborate multimodal courtship displays that are characterized by singing and simultaneous visual displays, 
such as holding nest material and bobbing (Goodwin 1982). We previously discovered that their visual courtship display includes multiple rapid steps while bobbing and produces non-vocal sounds (Ota et al. 2015; Figure 1(c); Supplementary Video 1). High-speed video observation revealed that they stamp their feet several times rapidly. The behaviour is invisible to the naked eye and appears as a single pulse on the spectrogram (Ota et al. 2015; Figure 1(a) and (b)). Our previous findings suggest that performing rapid step behaviour enables male and female cordon- bleus to communicate via multiple acoustic signals (i.e. vocal and non-vocal sounds).

Clark (2016) proposed that one criterion to diagnose a non-vocal sound is voluntariness: whether the sound is produced intentionally and production is modulated by the animal. Our previous study revealed that their tap dance-like behaviour is performed only during courtship, and the performance is adjusted in a context-dependent manner (Ota et al. 2015). For example, they took more steps when their partner stayed nearby and fewer steps when they were singing (Ota et al. 2015). These context-dependent performance changes seem to modulate non-vocal sounds but the relationship between the kinematics and sounds remains unclear. Investigating this relationship would improve our understanding of the role of non-vocal sounds.

In this study, we investigated whether taking multiple steps contributed to producing non-vocal sounds in two ways. First, we quantified dance performances by evaluating the number of steps in one bob and by evaluating step speed, and investigated how the dance performances affected sound amplitude. We predicted that if taking multiple steps plays an important role in producing non-vocal sounds, the step sound would be louder as the number of steps increased. We also expected that step speed (i.e. the number of steps/bobbing duration) would have an influence on step sound amplitude, because step speed would be associated with the velocity and/or force when their feet land on the perch. Second, we compared step sound amplitude with that of feet movement sounds in a non-courtship context and song notes to assess the efficacy of step sounds as acoustic signals. If they produce step sounds as acoustic signals, the sound would be louder than non-communicative sounds, such as feet movement sounds in a non-courtship context (Supplementary Video 2), and the amplitude would overlap with that of communicative vocal sounds (i.e. song notes). 


\section{Materials and methods}

\section{Subjects and experimental procedure}

We analysed courtship dances of 12 male and five female adult blue-capped cordon-bleus (>6 months old). One male and one female were arbitrarily paired and housed together in a cage $(120 \times 48 \times 48 \mathrm{~cm})$ in a sound-proof chamber. Their behaviours were recorded in a 2-h observational session with normal (Q3HD ZOOM, Tokyo, Japan; NTSC, 30 frames/s) and high-speed (GC-PX1 Victor, Yokohama, Japan; 300 frames/s) video cameras. Procedures were in accordance with German National Laws and approved by the Government of Upper Bavaria.

\section{Sound analysis}

We collected sound data with a sampling rate of $44.1 \mathrm{kHz}$ and 16-bit resolution, which was recorded with a unidirectional microphone (angle: $120^{\circ}$, max SPL: $130 \mathrm{~dB}$ ) under fixed gain settings in a normal-speed camera. We sampled on average, 41.1 step sounds from each individual (range, 13-50). We sampled step sounds that did not include any noise detected above background levels and were only produced on the same perch position in one session to minimize sound amplitude changes related to the distance of the birds to microphone (Figure 1(a); Supplementary Video 1). We also sampled 10-feet movement sounds that are not step sounds from each individual as controls (Figure 1(a)). We sampled the movement sounds when the birds moved on the perch where they also danced (Supplementary Video 2). In addition, 20 song notes that did not overlap with step sounds or other noises were arbitrarily sampled. We could not sample song notes from two females because they did not sing (see also Supplementary Figure S2).

We measured average and peak amplitude of step sounds, feet movement sounds, and song notes using Raven Pro 1.4 (www.birds.cornell.edu/raven). The durations of step sounds were also measured to quantify step speed (see 'Behavioural analysis'). Average amplitude was based on average values of sound pressure level during sound production (Charif et al. 2010). Peak amplitude was considered the sound pressure level at the darkest point in the sound spectrogram (Charif et al. 2010). Because cordon-bleus usually sing songs with 10-30 note types (Geberzahn and Gahr 2011) that have quite different amplitudes, their songs cover a wide range of amplitude (Figure 1(a)). To compare step sound amplitude with that of the lower limit of song 
notes, we selected the three lowest-amplitude song notes from the 20 song notes mentioned above.

Sound amplitude values measured in this study were expressed in decibels, which reflected relative values within each recording (Charif et al. 2010). The distances from microphones to birds $(67-76 \mathrm{~cm})$ and positions on the perch (i.e. edge or centre) differed among individuals, although we controlled for these issues at the within-individual level as described above. For these reasons, we could not investigate sound amplitude differences at an among-individual level in this study, but focused on the within-individual changes and statistically controlled for these changes (see also 'Statistical analysis').

\section{Behavioural analysis}

We analysed two candidate factors that can affect step sound amplitude: the number of steps in one bob and step speed index. From the high-speed movie images, we counted the number of steps in one bob that were analysed in sound analysis (Figure 1(a) and (c); Supplementary Video 1). As a variable that is independent of the number of steps, we calculated 'step speed index' (Supplementary Figure S1); we plotted the step sound duration against the number of steps and calculated the distance of each residual from the regression line (Supplementary Figure S1). A higher step speed index value indicates less time required for a particular number of steps (Supplementary Figure S1).

\section{Statistical analysis}

To assess whether dance performances influence sound amplitude, we investigated the effects of the number of steps and step speed index on sound amplitude using linear mixed-effect models (LME). To compare sound amplitude between sound categories (i.e. step sounds, feet movement sounds, and song notes), we investigated the effect of each sound category on sound amplitude using LME. We also compared amplitude between the lowest-amplitude song notes and step sounds using LME. In these analyses, we considered bird ID as a random effect to control for non-independence of data from the same individual. All statistical analyses were performed using $\mathrm{R} 3.1 .2$ ( $\mathrm{R}$ Development Core Team 2014). 


\section{Results}

Both average and peak sound amplitude increased as the number of steps increased (Figure 2, Table 1), which was also clear at the within-individual level (Supplementary Figure S2). Increased step speed index values negatively affected peak sound amplitude, but not average sound amplitude (Table 1).

Step sounds were significantly louder than feet movement sounds but quieter than song notes (Supplementary Video 2; Figure 2; average amplitude: $\mathrm{df}=1149, \mathrm{~F}=$ 970.943, $\mathrm{p}<0.001$; peak amplitude: $\mathrm{df}=1149, \mathrm{~F}=644.887, \mathrm{p}<0.001)$. Although amplitude of arbitrarily chosen song notes was clearly higher than step sound amplitude (Figure 2), the significant difference disappeared when we compared step sound amplitude with that of the lowest-amplitude song notes (Figure 2; average amplitude: df $=725, \mathrm{~F}=2.765, \mathrm{p}=0.097$; peak amplitude: $\mathrm{df}=725, \mathrm{~F}=1.702, \mathrm{p}=0.193)$.

\section{Discussion}

Our results showed that step sounds satisfy the criterion of non-vocal sounds as an acoustic signal (Clark 2016), at least under our captive conditions. As predicted, non-vocal sounds changed depending on the dance performances at the within-individual level. This suggests that context-dependent changes of dance performance (Ota et al. 2015) modulate the non-vocal sounds. Step sound amplitude correlated with the number of steps in one bobbing and taking multiple steps played an important role in producing louder sounds (Figure 2, Table 1). Additionally, step speed index had a negative effect on step sound amplitude (Table 1).

There are two possible mechanisms that explain these sound amplitude changes. First, the sound amplitude may be amplified by overlap of multiple step sounds. This may not be the case, however, because the experiments were conducted in a sound-proof chamber where sound reflections were substantially reduced. Our behavioural observations revealed that these birds usually tap their feet alternately (Figure 1(a), Supplementary Video 1) and rarely tap simultaneously (6.4\% of all bobbing actions in this study), which indicates that the tap dancing is not performed to make overlapping sounds. In addition, their step speed negatively affected peak sound amplitude (Table 1(b)). This finding indicates that higher step speed can increase the amount of overlap between consecutive sounds, but did not produce louder sounds. However, we should be cautious about the interpretation of these results, because it is 
not clear how step sounds are affected by reverberations in the eco-acoustic environment of the natural habitat of blue-capped cordon-bleus.

The second mechanism is strong tapping of their feet against the perch when they take certain steps. Considering that step speed negatively affected peak but not average sound amplitude, birds may more strongly tap during one step among the multiple steps at the cost of step speed. Taking multiple steps may positively increase the landing velocity of their feet on the perch, but we could not directly measure the landing velocity, force and the interval durations between taps, because neither image nor time resolution of our cameras were sufficient (Supplementary Video 1).

Our results of the comparison among sounds that the cordon-bleus produce in various contexts also support the idea that step sounds are not just by-product sounds of movements. Step sounds were distinct from other feet movement sounds (Supplementary Video 2; Figure 2) and were as loud as the lowest-amplitude song notes (Figure 2). Presumably, step sounds may serve as communicative signals, especially when the distance between birds is short.

Although we have revealed an interesting phenomenon about non-vocal sounds in songbirds, detailed communicative functions and the underlying physiological and neuromuscular mechanisms of these non-vocal sounds remain unclear. We cannot completely deny the possibility that the non-vocal sounds are just a by-product of a vigorous motion that is used as a visual signal. We should examine how signal receivers respond to non-vocal sounds (e.g. Freeman and Hare 2015). Perch choice of signal senders should also be tested, because perch structures or materials would affect acoustic features of non-vocal sounds. Investigating kinematic characteristics of the stepping (e.g. Clifton et al. 2015) and associated morphological features (e.g. Bostwick et al. 2012; Clark and Prum 2015) would further elucidate the mechanisms of non-vocal sounds in blue-capped cordon-bleus. Observing cordon-bleu courtship behaviour in the wild would help elucidate the eco-acoustic environment and perch properties that affect the production of these sounds. Such additional studies would shed new light on acoustic communication in birds. 


\section{Acknowledgements}

We are grateful to Dr. Albertine Leitão for help with the experiments. We also thank the Max Planck Institute for Ornithology staff for providing the experimental equipment and maintaining the birds.

\section{Funding}

This work was supported by JSPS Grants-in-Aid for Young Scientists under grants 22800002 and 23680027 to MS; and a Grant-in-Aid for JSPS Fellows under grant $15 \mathrm{~J} 02516$ to NO.

\section{References}

Andersson M. 1994. Sexual selection. Princeton: Princeton University Press.

Bahr PH. 1907. On the "bleating" or "drumming" of the snipe (Gallinago coelestis). Proc. Zool. Soc. Lond. 77:12-35.

Bostwick KS, Prum RO. 2003. High-speed video analysis of wing-snapping in two manakin clades (Pipridae: Aves). J Exp Biol. 206:3693-3706.

Bostwick KS, Riccio ML, Humphries JM. 2012. Massive, solidified bone in the wing of a volant courting bird. Biol Lett. 8:760-763.

Byers J, Hebets E, Podos J. 2010. Female mate choice based upon male motor performance. Anim Behav. 79:771-778.

Catchpole CK, Slater PJB. 2008. Bird song: biological themes and variations. $2^{\text {nd }}$ ed. Cambridge: Cambridge University Press.

Charif RA, Waack AM, Strickman LM. 2010. Raven Pro 1.4 User's Manual. Ithaca: Cornell Laboratory of Ornithology.

Clark CJ. 2016. Locomotion-induced sounds and sonations. mechanisms, communication function, and relationship with behavior. In: Suthers RA, Fitch WT, Fay RR, Popper AN, editor. Vertebrate sound production and acoustic communication. Switzerland: Springer International Publishing; p. 83-117.

Clark CJ, Elias DO, Prum RO. 2011. Aeroelastic flutter produces hummingbird feather 
songs. Science. 333:1430-1433.

Clark CJ, Prum RO. 2015. Aeroelastic flutter of feathers, flight and the evolution of non-vocal communication in birds. J Exp Biol. 218:3520-3527.

Clifton GT, Hedrick TL, Biewener AA. 2015. Western and Clark's grebes use novel strategies for running on water. J Exp Biol. 218:1235-1243.

Eda-fujiwara H, Yamamoto A, Sugita H, Takahashi Y, Kojima Y, Sakashita R, Ogawa H, Miyamoto T, Kimura T. 2004. Sexual dimorphism of acoustic signals in the Oriental white stork : non-invasive identification of sex in birds. Zoolog Sci. 21:817-821.

Freeman AR, Hare JF. 2015. Infrasound in mating displays : a peacock's tale. Anim Behav. 102:241-250.

Fusani L, Giordano M, Day LB, Schlinger BA. 2007. High-speed video analysis reveals individual variability in the courtship displays of male golden-collared manakins. Ethology. 113:964-972.

Fusani L, Schlinger BA. 2012. Proximate and ultimate causes of male courtship behavior in Golden-collared manakins. J Ornithol. 153:S119-S124.

Garcia M, Charrier I, Rendall D, Iwaniuk AN. 2012. Temporal and spectral analyses reveal individual variation in a non-vocal acoustic display: The drumming display of the ruffed grouse (Bonasa umbellus, L.). Ethology. 118:292-301.

Geberzahn N, Gahr M. 2011. Undirected (solitary) birdsong in female and male blue-capped cordon-bleus (Uraeginthus cyanocephalus) and its endocrine correlates. PLoS One. 6:e26485.

Goodwin D. 1982. Estrildid finches of the world. Ithaca: Cornell University Press.

Koch RE, Krakauer AH, Patricelli GL. 2015. Investigating female mate choice for mechanical sounds in the male Greater sage-grouse. Auk. 132:349-358.

Norberg RA. 1991. The flappet lark Mirafra rufocinnamomea doubles its wingbeat rate to $24 \mathrm{~Hz}$ in wing-clap display: a sexually selected feat. J Exp Biol. 159:515-523.

Odom KJ, Hall ML, Riebel K, Omland KE, Langmore NE. 2014. Female song is widespread and ancestral in songbirds. Nat Commun. 5:3379. 
Ota N, Gahr M, Soma M. 2015. Tap dancing birds: the multimodal mutual courtship display of males and females in a socially monogamous songbird. Sci Rep. 5:16614.

Prum RO. 1998. Sexual selection and the evolution of mechanical sound production in manakins (Aves : Pipridae). Anim Behav. 55:977-994.

R Development Core Team. 2014. R: A language and environment for statistical computing. Vienna: R Foundation for Statistical Computing.

Soma M, Mori C. 2015. The songbird as a percussionist: syntactic rules for non-vocal sound and song production in Java sparrows. PLoS ONE. 10(5):e0124876

Stark RD, Dodenhoff DJ, Johnson EV. 1998. A quantitative analysis of woodpecker drumming. Condor. 100:350-356.

Wood GA. 1987. Further field observations of the palm cockatoo Probosciger aterrimus in the Cape York Peninsula, Queensland. Corella. 12:48-52. 


\section{Figures \& Tables}

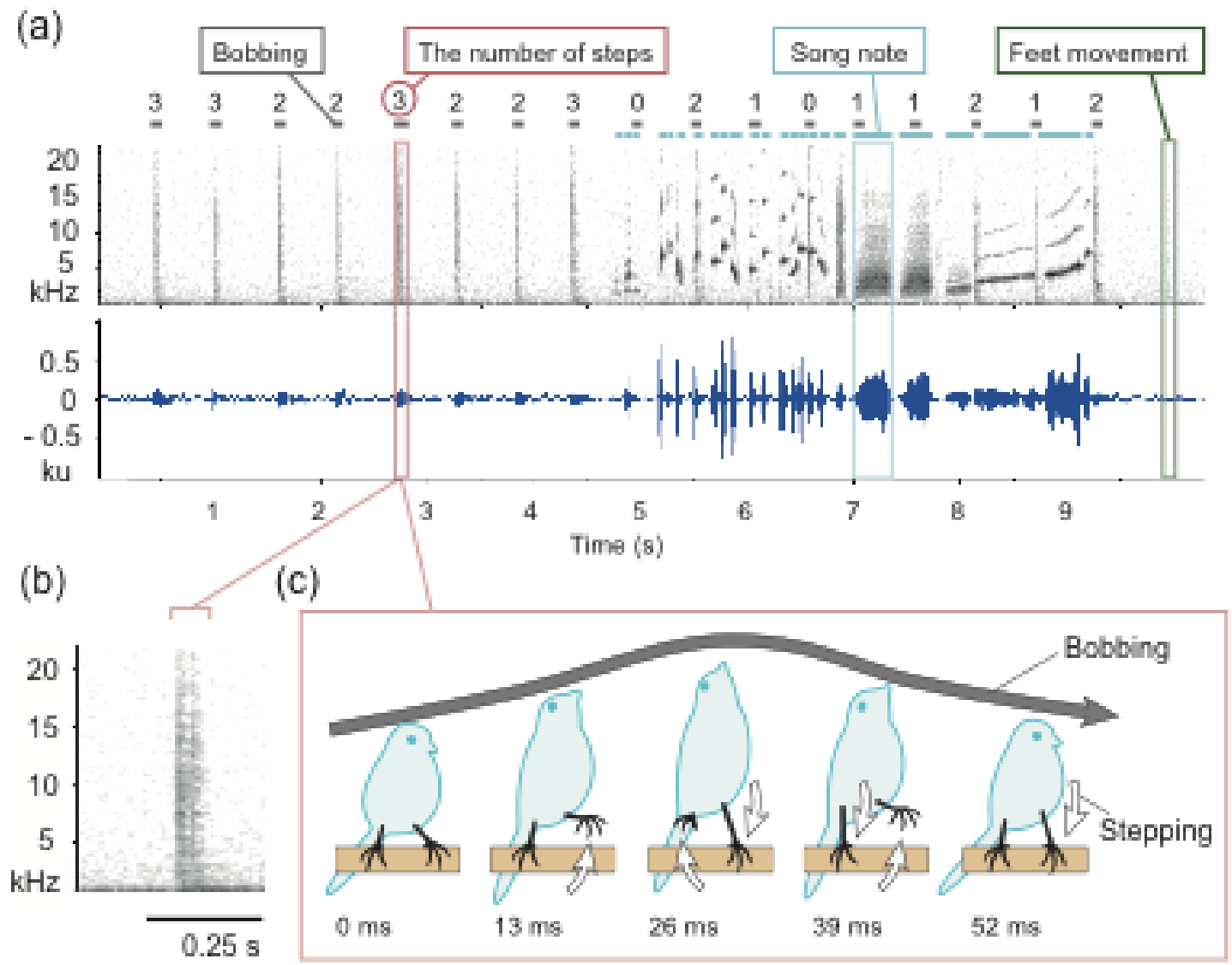

Figure 1. Blue-capped cordon-bleu step behaviour and sounds. (a) A spectrogram and waveform of blue-capped cordon-bleu step sounds and song. These birds simultaneously bob and step, and sing at certain times. Numbers at the top of the spectrogram indicate how many steps were performed to produce the sounds. Feet movement sounds and song notes were arbitrarily sampled from each individual. (b) Enlarged view of the step sound spectrogram. (c) Diagram of step behaviour that produces non-vocal sounds. 

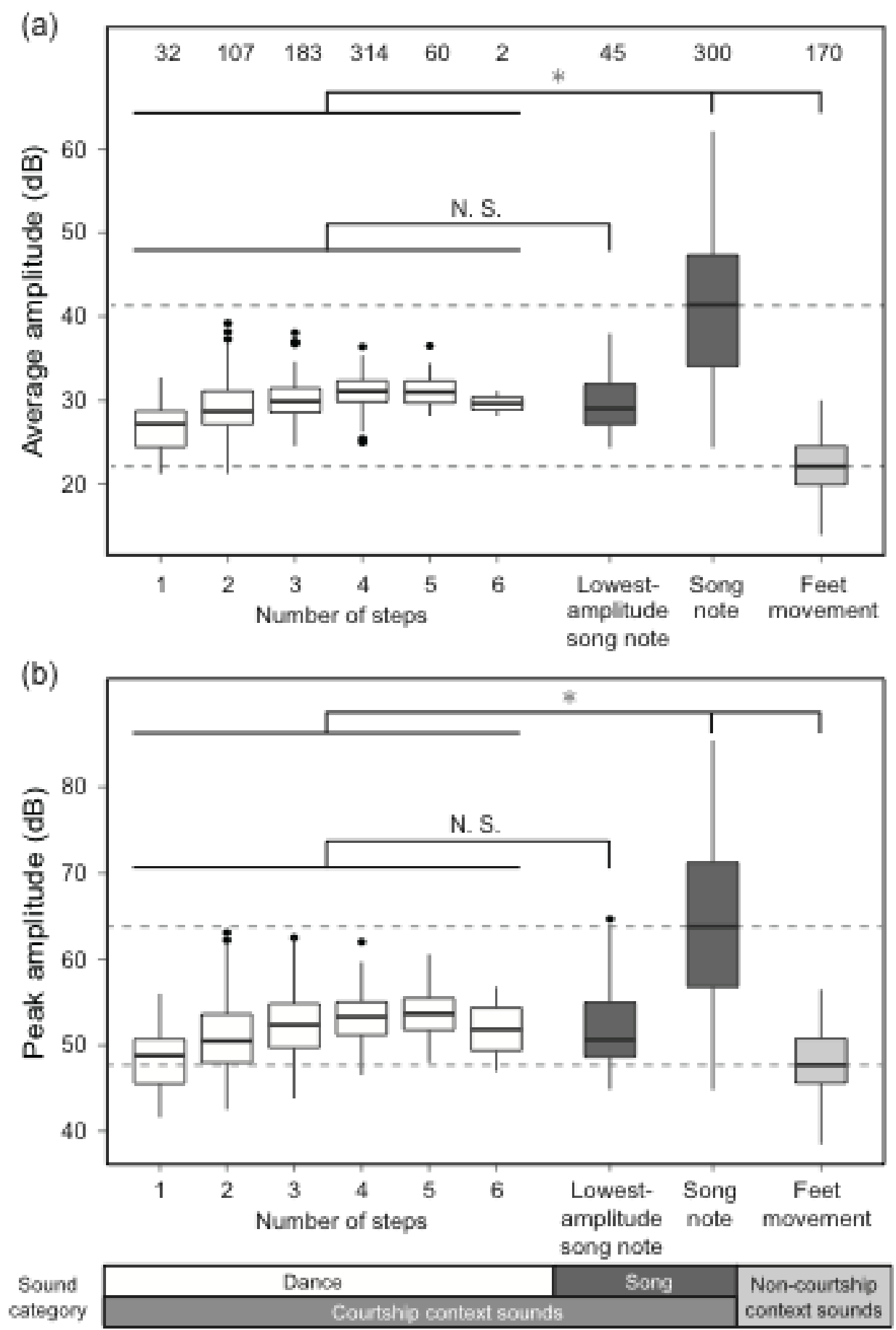

Figure 2. Effects of the number of steps on sound amplitude. (a) Average and (b) peak sound amplitude are plotted as a function of the number of steps compared with those of lowest-amplitude song notes, arbitrarily chosen song notes, and feet movement sounds. Numbers at the top of (a) represent sample size for both (a) and (b) (see also Supplementary Figure S2). All box plots show median and quartiles. Outliers are plotted as points. 
Table 1. Results of LME analyses of step sound amplitude. The effects of the number of steps and step speed index on (a) average and (b) peak step sound amplitude.

\begin{tabular}{|l|l|r|r|r|r|}
\hline Response variable & Independent variable & Coefficient & SE & t & $P$ \\
\hline (a) Average amplitude & The number of steps & 0.928 & 0.090 & 10.307 & $<\mathbf{0 . 0 0 1}$ \\
\cline { 2 - 6 } & Step speed index & 4.578 & 6.458 & 0.709 & 0.479 \\
\hline (b) Peak amplitude & The number of steps & 1.241 & 0.129 & 9.704 & $<\mathbf{0 . 0 0 1}$ \\
\cline { 2 - 6 } & Step speed index & -42.001 & 9.191 & 4.571 & $<\mathbf{0 . 0 0 1}$ \\
\hline
\end{tabular}

